\title{
The Conference "Analytical Methods of Celestial Mechanics" and Celebration of the 300th Anniversary of L. Euler's Birth in St. Petersburg
}

\author{
A. D. Bryuno, N. N. Vasil'ev, V. V. Orlov, and K. V. Kholshevnikov
}

DOI: $10.1134 / \mathrm{S} 0038094609010018$

April 15, 2007 marked the 300th anniversary of the birth of Leonhard Euler, one of the greatest mathematicians, mechanics, and astronomers. In light of the anniversary, in summer 2007, the International Euler Mathematical Congress dedicated to this memorable date was held in St. Petersburg. The Congress's central event was the "Euler Festival" with plenary presentations by the most well-known mathematicians such as G. Wustholz, V. Zakharov, V.V. Kozlov, A. Connes, L. Lovasz, Yu.I. Manin, S.P. Novikov, L.D. Faddeev, and F. Hirzebruch.

In addition, within the framework of the Congress, there were nine specialized conferences dedicated to individual fields of mathematics, mechanics, and physics where L. Euler's contribution was the most significant. One of these conferences-the conference "Analytical Methods of Celestial Mechanics"-was held on July 8-12, 2007 at the Euler International Mathematical Institute. About 60 specialists from Russia, France, Spain, Poland, Finland, Germany, Romania, and Ukraine gathered for this event. One should emphasize that the roots of analytical, numerical, and qualitative methods of theoretical and applied celestial mechanics, including descriptions of the motion of the Solar System's natural and artificial bodies, come from the works by Leonhard Euler.
The presentation themes included analytical and qualitative methods, Hamiltonian mechanics, the threebody problem, stellar dynamics, planet and sputnik motion in the Solar System, and the interaction of numerical and analytical methods. Since its origin, stellar mechanics has been closely connected with observations of celestial bodies and numerical calculations. Moreover, some numerical methods were developed specifically for this field. At present, celestial mechanics also applies and develops various systems of analytical calculations.

From the standpoint of today, the main classical analytical methods of celestial mechanics can be regarded as regular perturbation methods, such as averaging, normal form, etc. Although there has been a significant spurt in the development of the primary regular perturbation methods since the time of L. Euler, there are still a number of specific unresolved tasks. Some classical problems of celestial mechanics (for example, the description of combustions and Hill's problem) can be regarded as problems with singular perturbations. Nowadays, these are solved by means of regularization and desingularization.

Some of the above listed aspects of celestial mechanics were presented at the conference. In the present issue, we publish papers related to the description of the motion of the Solar System bodies. 